\title{
Spatial Information Grid-based Digital Map Framing Method
}

\author{
Guiling $\mathrm{Li}^{1, \mathrm{a}^{*}}$, Yongchong Yang ${ }^{2, \mathrm{~b}}$ \\ ${ }^{1}$ College of Architecture \&Civil Engineering, Jiaxing University, China \\ ${ }^{2}$ College of Surveying Science and Technology, Xi'an University of Science and Technology, China \\ agljx@126.com, byang_yongch@163.com
}

Keywords: Digital map, map framing, spatial information grid, grid, block.

Abstract: In view of the defects of traditional map framing methods, a new map framing method, not only is which suitable for digital environment but also does that consider the natural features and social characteristics of earth space, is put forward. The resolution and the amount of geographic information are dependent on the size of blocks, of which each geographical entity has an administrative code and property ID according to the principle of keeping integrity of features and regions. That digital map framing method can divide the geographic area into geospatial cells, one of which records and stores its various basic geographical elements and sets up its spatial cell file according to an administrative system or natural zone. The geospatial division and the spatial data organization method provide convenience for the spatial index and make the spatial statistics be more convenient. It is a division method of spatial location, and is also the information carrier of natural, social and economic properties for a particular spatial location. At the same time, it is a novel expression method suitable for spatial information changing with space time coordinates in order to realize the integration, sharing and utilization of spatial information resources under the grid computing environment.

\section{Disadvantages of digital map framing}

After the digitized surveying technologies have widely been used, the digital map illustrated with points, lines, faces and entities bunched through discrete points appeared. Most of digital maps can still be managed and preserved in terms of framing because the stipulation of map framing still abides by the requirements of surveying and mapping paper maps in corresponding regulations of map surveying and mapping. Therefore, current digital maps can only be utilized after they are merged, too. Nevertheless, whether or not does the digital world need map framing becomes a controversial issue after the traditional simulating map becomes a digitized product.

The application of digital map is different from the use of paper maps. The size of a map is only restricted to computer data treatment and graphic display capacity when the research range enlarges under a digital condition. There is a great disparity between the application of paper maps and the usage of digital maps whether viewing maps or amount of computing, this is mainly because those maps have accomplished the automation of map utilization, namely, the application of all the maps are finished through a computer program under human-computer interaction. Firstly, they can continuously be viewed in a broad range, and can be watched in a magnified fashion, too. Thus, one can browse an arbitrary district map, and one may look at any range or dimension map by means of an arbitrary scale. Secondly, they can automatically measure and calculate the length and area of features, and have automatic analysis functions like the geographic information system (GIS). But those functions have still proposed much higher requirements.

Not only does the framing of digital maps destroy the integrity of a district, but also does it break the integrity of many features (mainly for area- and line-type features). The framing of digital maps can make a spatial object become objected and divide an entity through framing in different 
kinds of discontinuous maps. An after-divided entity with its self spatial data enters an isolated space system, this gives rise to a complicated map matching problem [1]. Consequently, a great deal of digital map utility software is required to have an automatically seamless splice function for framing maps.

There is a physically seamless technology and a seamlessness-viewed one for digital framing maps. So called seamlessness-viewed technology is a kind of technological treatment by which those maps are just looked to be seamless on a screen. Not only does an authentic seamlessness need to look seamless, but also does it continue from any direction in the sense of true topology and precisely match each other on multi-resolution in order to reach unity measuring and analysis.

But all above, in the condition of traditional technologies the framing of paper map is necessary, but under digital condition the framing of digital map is useless, evenly harmful. It can be seen from the requirements of digital earth to digital city and their applications that those need people break through the frame of geospatial data and information illustrated by means of the framing of map existing for several thousand of years to seek a new expression methodology for the geospatial data and information under digital environment and create a new expression method for the spatial data suitable for network computing all over the world.

\section{Map blocking methodology based on anomaly grid}

\section{Spatial information grid}

The spatial information grid is a systematic organization of spatial information, or can be understood as a systematic organization of spatial information from geo-science. In general, if the logical model of spatial data in a spatial data model organizes data with an object entity as an element according to logical theory, the spatial information grid will be an integrated organization for whole geospatial entity information, which can be seamless and overlay-free in a view to space from overall to local and top to bottom, and has a systematic structure and integrity $[1,4]$.

At present there are two division methods: regular and irregular grid methods for spatial information grids. The regular grid method means that the whole world and nation range will be divided into different crude or fine level grids (for instance, four level grid) according to various longitude and latitude grid size, each level grid possesses a upper-lower coverage relationship up to extent. The irregular grid method indicates that the dissimilar level grids will build up a connection with the administration levels of the whole nation, province, region (city) and county and so on, at the same time set up a systematic structure of our spatial information multi-grid (SIMG) uniting the construction of our national and provincial-lever spatial information infrastructures and information network technology.

\section{Basic idea of digital map blocking}

In essence, the blocking of digital map is still an organization of geospatial division and spatial data. Thus, it is necessary to carry out research for uniting spatial information grid and the blocking of digital map.

Geographic information has to be expressed in a finite framing plane for paper-type map, whose mathematical basis mainly resides in the coordinate grid of established map, and fills context of map as a framing. In consequence, the framing of map is necessary for paper-type map. However, digital map can express geographic information in a broadly mathematical space, the action of its mathematical basis mainly resides in making each point position of digital map element locate at a basis reference or coordinate system, namely, wholly show all the geographic information in a cartographic area in order to avoid map-sheet matching and coordinate transfer. But the size of digital map is limited to computer data treatment and graphical display capacity, and it still needs to 
be measured and mapped, stored and utilized in a blocking way.

For this purpose, we put forward a novel framing methodology of digital map based on an irregular grid, by means of which a cartographic district is divided into many blocks according to physical geography layout and administrative division actuality. The core idea of this method abides by the principle of keeping the integrity of features and regions, and determines the size of block according to measuring scale and amount of geographical information in a region. Firstly, the method needs to determine the size of a spatial element called as a resolution of geographical analysis and yet a leading indicator of cartographic accuracy. Secondly, each geographic entity has an administrative division code property and identification number in a block, namely, an ID number similar to human being'. This property provides convenience for spatial position and indexing, especially spatial design.

Based on the abovementioned idea, the whole nation range will be subdivided into various course- and fine-level grids by means of dissimilar district size, each level grid possesses a coverage relationship between upper and lower stratum up to extent. Those irregular-level grids are parallel to dissimilar time and space scales, any point can be corresponding to a set of appropriate multi-level grid element through endowing an identifier (coding) to the irregular grid system, in other words, any point can be positioned by the identifier (geographical code) [7].The size (layered density) of required grid can be determined according to the concentrated extent of practical features when partitioning mesh, for example, the place where features are sparse only requires course-level grids, and the place (city) where features are highly concentrated must store spatial and non-spatial data by means of fine-level grids. Different level grids will be set up correlations with the whole nation, province, region (city) and county and so on, while the system structure of Chinese framing (blocking) of digital map will be set up uniting the construction and information internet technology of our nation and province-level spatial information infrastructure.

A novel methodology to the framing of digital map is a division one of spatial district, and is a spatial positioning one, too. The grid determined by the blocking of digital map is a reference system of ID-based indirect spatial, grid and its geo-code are dual relations where the grid can precisely limit the range corresponding to its geo-code, which means the identifier of the grid cell. It is an information carrier on nature, society and economy property for an especial range of spatial location, the city spatial information resource can be integrated using an object-oriented theory and a unique identifier of spatial object with geo-code [7]. While it is also a new expression way to spatial information changing with time-space coordinate system in order to conveniently accomplish the integration, sharing and utilization of spatial information resource under grid calculation environment.

\section{Basic principle of digital map blocking}

The blocking of digital map should be a polygon real region with legible boundaries in terms of certain principles. Following principles comprehensively considered are:

1) Legal basis principle: the division of block can be carried out in terms of legally administrative boundaries.

2) Territorial management principle: the maximal boundary of block defines one of the administrative region, which does not bridge the segmentation of the administrative region.

3) Geographic layout principle: geographic layout such as street, yard, common lawn, plaza, bridge, clearing, river, mountain, lake and so on, should naturally be divided under a minimally administrative region.

4) Load balance principle: the boundaries of block should not pass through its features and regions while the number of features in each block should be roughly equal considering integrity of features and regions. 
5) Seamless joint principle: there should not be leak and overlay between two adjacent blocks.

6) Relatively stable principle: the segmentation of block should maintain relatively stable.

\section{Division method of digital geographic block}

The blocking of digital map should be in agreement with spatial information grid. The size of block is dependent on the quantity of blocks, and its range is dependent on its administrative or natural or

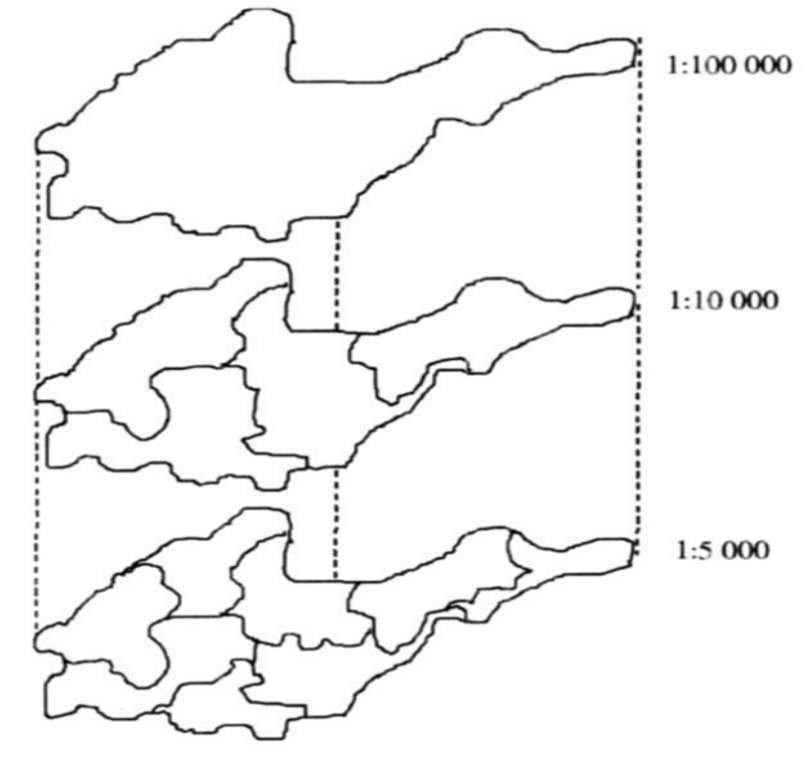

Figure 1 se gme ntation system of digita1 Topographic map for a medium- leve 1 city professional region in order to make the best of avoiding destroying features and administrative regions. For instance, for large-scale digital geographic map of city or town, street is considered as a basic block, however, for small-scale digital geographic map of village or field, village- and town-level administrative region is viewed as a basic block. We have designed a blocking system of digital topographic map for a medium-sized city as shown in Fig.1 according to the abovementioned blocking principle.

Different from a paper-type map of standardized framing, the blocking of digital map does not have its profile. Therefore, it reflects its basic information using header file where the form of header file is listed in

Table. 1 only by means of digital image. The header file is named in terms of map name or map framing number of digital map block. In a word, the header file name should be in good line with its digital map block name where its format can be in text fashion, and can yet be in electrical table way.

Table 1 header file of digital map block

\begin{tabular}{ll}
\hline Product title & Digital geographic map \\
\hline Map title & Culture Park \\
Number of framing & 4251260203 \\
Measuring scale & 1000 \\
Property Unit & XX city bureau of planning \\
Surveying and mapping unit & XX city institute of surveying \& mapping \\
Coordinate system & XX city coordinate system \\
Elevation system & 1985 national elevation benchmarks \\
Date of surveying and mapping & July,2007 \\
Method of mapping & Outdoor digitalized surveying and mapping \\
Remarks & \\
\hline
\end{tabular}

\section{Encoding principle of blocks and features}

A block should have a unique code in the sense of time and space and entitle the bigger geographic name or organization name in blocks. Its former code should not be occupied and the recently added block should carry out extension according to the former code principle when the block is 
modified. The coding of block or the number of map consists of ten number, of which former six number stands for the administrative region code of county-level or above county-level, in turn two number indicates the code of village or town, latter two number means the sequential code of block, which sorts in a village or town code from left to right and upper to bottom. When an administrative region above county-level is taken as a block, both of village and town (street) code and the sequential segment code will be encoded as 00 , by parity of reasoning, when village and town (street) region is viewed as block, the sequential segment code will be encoded as 00 .

The identification code of features composes of eight number, of which former four number (where former and latter two number defines the large and small-classification code of features ,respectively ) stands for the classification code of features, in turn latter four number means the sequential code of similar features. When a kind of feature in a block is only one, the sequential code of the feature is coded as 0001 . When the plotting scale of map is relatively tiny, the classification code of feature can only use the big classification code.

\section{Summary}

In essence, the blocking of digital map is still an organization of geospatial division and spatial data. The blocking method of digital map proposed by us, which breaks through the traditional habit of formerly trapezoid and rectangular framing, is a novel framing one appropriate to not only digital environment but also natural characteristics and social properties. It will divide a geographic region into geospatial elements in terms of an administrative district, each spatial element records and stores dissimilarly basic geographic elements, and builds up its geographic data file. Not only is this novel method to subdivide a digital map greatly convenient for surveying, cartography and application of current digital map, but also is it important for investigation of spatial information grid.

\section{Acknowledgements}

This work was financially supported by the Science and Technology Project of Jiaxing City, Zhejiang Province, China (No.2013AY11018)

\section{References}

[1] P.Hu, L.You,H.Hu, the Outline of Map Algebra, Surveying and Mapping Press, Beijing ,2008.

[2] D.R.Li: submitted to Journal of Remote Sensing (2005)

[3] Y.Liu, C.M.Li,D.Q.Liu, W.J.Ma: submitted to Science of Surveying and Mapping (2007)

[4] D.R.Li, X.Y.Zhu, J.Y.Gong: submitted to Geomatics and Information Science of Wuhan University (2003)

[5] G.X.Chen,D.Zhang,D.Zhang, H.Y.Zhou,et.al :submitted to Science of Surveying and Mapping(2005)

[6] G.L: submitted to Journal of WUT (Information \& Management Engineering) (2005)

[7] J.Li,Z.Q.Luo,L.Hao, Z.Z.An,et.al: submitted to Geomatics and Information Science of Wuhan University (2005)

[8] D.R.Li, Z.F.Xiao, X.Y.Zhu, J.Y.Gong, et.al:submitted to ACTA GEODAETICA et CARTOGRAPHICA SINICA(2006) 